Pacific Journal of Mathematics

ON EQUIVALENCE OF GAUSSIAN MEASURES 


\title{
ON EQUIVALENCE OF GAUSSIAN MEASURES
}

\author{
DALE E. VARBERG
}

1. Introduction. When are two Gaussian processes equivalent (mutually absolutely continuous with respect to each other)? More precisely, given $\left\{S, B, m_{i}\right\}, i=1,2$, where $S$ is a set of real valued functions on some interval $[a, b], B$ is a Borel field of subsets of $S$ and $m_{i}$ is a Gaussian probability measure on $B$, under what conditions is $m_{1}$ equivalent to $m_{2}$ ? This question has been investigated, by several authors. In particular, we mention Jacob Feldman, who in a recent paper [5] has shown that a certain dichotomy exists. If $S$ is a linear space, then either $m_{1}$ and $m_{2}$ are equivalent or they are perpendicular (mutually singular). Moreover, using some results of Segal [6], he has shown that, if $K$ is the linear span of $S$ and the real constants, then $m_{1}$ and $m_{2}$ are equivalent if and only if the $m_{1}$-equivalence classes of $K$ are the same as the $m_{2}$-equivalence classes of $K$ and the identity correspondence between the $L_{2}\left(m_{1}\right)$ closure of $K$ and the $L_{2}\left(m_{2}\right)$ closure of $K$ is a bounded invertible operator $T$ such that $\left(T^{*} T\right)^{1 / 2}-I$ is a Hilbert Schmidt operator.

We propose to look at this question from a somewhat different point of view. It is well known that a Gaussian process and hence its probability measure is determined by a covariance function $r(s, t)^{1}$. It should therefore be possible to answer the question posed above directly in terms of conditions on the convariance functions of the two processes. We are able to do this for a rather wide class of Gaussian Markov processes (Theorem 1), and we conjecture that an answer of this type is possible in general. The crucial condition appears to be that the first derivatives of the two covariance functions have the same jump on the diagonal $s=t$. To set the stage for our main theorem, we make the following definition.

Definition 1. Let $M \equiv M[a, b]$ denote the class of all Gaussian processes $\{x(t), a \leqq t \leqq b\}$ with mean function identically zero and covariance function $r(s, t)$ given by

$$
r(s, t)=\left\{\begin{array}{ll}
u(s) v(t) & s \leqq t \\
u(t) v(s) & s \geqq t
\end{array}\right\},
$$

where moreover,

(A) $u(a) \geqq 0$,

Received June 12, 1960.

1 More correctly, it is determined by a covariance function $r(s, t)$ and a mean function $m(t)$, (see [3], p. 72). We assume that the mean function is identically zero throughout this paper. 
(B) $v(t)>0$ on $[a, b]$,

(C) $u^{\prime \prime}$ and $v^{\prime \prime}$ exist and are continuous on $[a, b]$,

(D) $v(t) u^{\prime}(t)-u(t) v^{\prime}(t)>0$ on $[a, b]^{2}$.

Almost all sample functions of such processes are continuous (see [4], pp. 401, 402). We shall assume therefore that the space of sample functions of processes belonging to the class $M[a, b]$ is $\{C, B\}$ where $C \equiv C[a, b]$ is the set of all continuous real valued functions on $[a, b]$ and $B$ is the Borel field of subsets of $C$ generated by sets of the form $\left\{x \in C: a_{k}<x\left(t_{k}\right) \leqq b_{k}, k=1,2, \cdots, n, t_{k} \in[a, b]\right\}$.

THEOREM 1. Let $\{x(t), a \leqq t \leqq b\}$ and $\{y(t), a \leqq t \leqq b\}$ be two Gaussian processes belonging to $M[a, b]$ with probability measures $m_{r}$ and $m_{\rho}$ determined by their respective covariance functions $r(s, t)$ and $\rho(s, t)$. Let

$$
r(s, t)=\left\{\begin{array}{ll}
u(s) v(t) & s \leqq t \\
u(t) v(s) & s \geqq t
\end{array}\right\}, \quad \rho(s, t)=\left\{\begin{array}{ll}
\theta(s) \phi(t) & s \leqq t \\
\theta(t) \phi(s) & s \geqq t
\end{array}\right\} .
$$

Then, necessary and sufficient conditions that $m_{r}$ be equivalent to $m_{\rho}$ are that

(E) $v(t) u^{\prime}(t)-u(t) v^{\prime}(t)=\phi(t) \theta^{\prime}(t)-\theta(t) \phi^{\prime}(t)$ on $[a, b]$,

(F) $u(a)$ and $\theta(a)$ are either both zero or both non-zero.

Moreover, if these conditions are satisfied, the Radon-Nikodym derivative of $m_{\rho}$ with respect to $m_{r}$ is given by

$$
d m_{\rho} / d m_{r}=C_{1} \exp \left\{[1 / 2]\left[C_{2} x^{2}(\alpha)+\int_{a}^{b} f(t) d\left\{x^{2}(t) /[\phi(t) v(t)]\right\}\right]\right\},
$$

where

$$
\begin{aligned}
C_{1} & =\left\{\begin{array}{ll}
\{[\phi(a) v(b)] /[\phi(b) v(a)]\}^{1 / 2} & \text { if } \theta(a)=0 \\
\{[u(a) v(b)] /[\theta(a) \phi(b)]\}^{1 / 2} & \text { if } \theta(a) \neq 0
\end{array}\right\}, \\
C_{2} & =\left\{\begin{array}{ll}
0 & \text { if } \theta(a)=0 \\
{[\phi(a) \theta(a)-u(a) v(a)] /[v(a) \phi(a) \theta(a) u(a)]} & \text { if } \theta(a) \neq 0
\end{array}\right\}
\end{aligned}
$$

and

$$
f(t)=\left[v(t) \phi^{\prime}(t)-\phi(t) v^{\prime}(t)\right] /\left[v(t) u^{\prime}(t)-u(t) v^{\prime}(t)\right]^{3} .
$$

The "necessity" part of the proof depends on a theorem of Baxter while the "sufficiency" will be made to depend on several lemmas.

${ }^{2}$ Conditions (A), (B) and (D) insure that $r(s, t)$ is a covariance function. Covariance functions which factor this way are sometimes called triangular covariance functions. Gaussian processes determined by triangular covariance functions may be shown to be Markov processes.

${ }^{3}$ The corresponding theorem for the Wiener process on $[0,1]$, (i.e., for the case $r(s, t)=$ $\min (s, t)$ ), was obtained by a somewhat different method in the author's doctoral dissertaton written under the direction of Professor R. H. Cameron (see [8]). 
After proving the theorem, we give several examples, one of which (Example 3) implies a result previously obtained by Charlotte T. Striebel in connection with Ornstien Uhlenbeck processes.

\section{Baxter's theorem and a corollary.}

BAXTER'S THEOREM. Let $\{x(t), \alpha \leqq t \leqq \beta\}$ be a Gaussian process with mean function identically zero and continuous covariance function $r(s, t)$, $r$ having uniformly bounded second derivatives for $s \neq t$. Let

$$
f_{r}(t)=\lim _{s \rightarrow t} \frac{r(t, t)-r(s, t)}{t-s}-\lim _{s \rightarrow t} \frac{r(t, t)-r(s, t)}{t-s} .
$$

Then with probability one,

$$
\lim _{n \rightarrow \infty} \sum_{k=1}^{2^{n}}\left[x\left(t_{k}\right)-x\left(t_{k-1}\right)\right]^{2}=\int_{\alpha}^{\beta} f_{r}(u) d u
$$

where $t_{k}=\alpha+k(\beta-\alpha) 2^{-n}, k=0,1,2, \cdots, 2^{n 4}$.

COROLlaRY. Let $\{x(t), a \leqq t \leqq b\}$ and $\{y(t), a \leqq t \leqq b\}$ be Gaussian processes with mean functions identically zero and covariance functions $r(s, t)$ and $\rho(s, t)$ determining probability measures $m_{r}$ and $m_{\rho}$ respectively. Suppose that $r$ and $\rho$ satisfy the conditions of the above theorem. Then, if $m_{r}$ is equivalent to $m_{\rho}$, it follows that $f_{r}(t)=f_{\rho}(t)$ for all $t \in[a, b]$.

Proof of corollary. Let $S$ denote the common space of sample functions of the two processes and let

$$
N_{\beta}^{r}=\left\{x \in S: \lim _{n \rightarrow \infty} \sum_{k=1}^{2^{n}}\left[x\left(t_{k}\right)-x\left(t_{k-1}\right)\right]^{2}=\int_{a}^{\beta} f_{r}(u) d u\right\}
$$

where $t_{k}=a+k(\beta-a) 2^{-n}, k=0,1,2, \cdots, 2^{n}, \beta \in[a, b]$. By Baxter's theorem $m_{r}\left\{N_{\beta}^{r}\right\}=1=m_{\rho}\left\{N_{\beta}^{\rho}\right\}$. Now let $J$ denote the Radon-Nikodym derivative of $m_{\rho}$ with respect $m_{r}$. Then if $\chi$ denotes the set characteristic function of $N_{\beta}^{r}$, we have

$$
m_{\rho}\left\{N_{\beta}^{r}\right\}=E^{\rho}\{\chi(y)\}=E^{r}\{\chi(x) J(x)\}=1^{5} .
$$

Hence $m_{\rho}\left\{N_{\beta}^{r}\right\}=m_{\rho}\left\{N_{\beta}^{\rho}\right\}=1$, i.e., for each $\beta \in[a, b], N_{\beta}^{r}$ and $N_{\beta}^{\rho}$ are sets of $m_{\mathrm{\rho}}$ measure one. It follows that $\int_{a}^{\beta} f_{r}(u) d u=\int_{a}^{\beta} f_{\mathrm{p}}(u) d u$ for each $\beta \in[a, b]$ and, since $f_{r}$ and $f_{\rho}$ are continuous, $f_{r}(\beta)=f_{\mathrm{p}}(\beta)$ for each $\beta \in[a, b]$.

This result gives the "necessity" almost immediately (see $\S 5$ ). The

${ }_{4}$ This actually is a slight generalization of the theorem of Baxter (see [1]), the generalization being that we state the result $(2.0)$ for the interval $[\alpha, \beta]$ rather than $[0,1]$.

${ }^{5} E^{r}$ denotes expected value on the Gaussian process with covariance function $r$. 
"sufficiency" is apparently harder to demonstrate. To facilitate matters we introduce some notation and prove four lemmas.

3. Notation. The following notation will be used throughout the rest of this paper.

$$
\begin{aligned}
& r(s, t)=\left\{\begin{array}{ll}
u(s) v(t) & s \leqq t \\
u(t) v(s) & s \geqq t
\end{array}\right\}, \\
& \rho(s, t)=\left\{\begin{array}{ll}
\theta(s) \phi(t) & s \leqq t \\
\theta(t) \phi(s) & s \geqq t
\end{array}\right\},
\end{aligned}
$$

$r$ is the covariance function of the process $\{x(t), a \leqq t \leqq b\}$, $\rho$ is the covariance function of the process $\{y(t), a \leqq t \leqq b\}$,

$$
\begin{aligned}
w(s) & =v(s) u^{\prime}(s)-u(s) v^{\prime}(s), \\
\omega(s) & =\phi(s) \theta^{\prime}(s)-\theta(s) \phi^{\prime}(s), \\
f(s) & =\left[v(s) \phi^{\prime}(s)-\phi(s) v^{\prime}(s)\right] / w(s), \\
m & =2^{n}, \\
t_{k} & =a+k(b-a) / m, \quad k=0,1,2, \cdots, m ; n>0,
\end{aligned}
$$

for any function $g, g_{k}=g\left(t_{k}\right)$, unless otherwise indicated,

$$
\begin{aligned}
& \hat{w}_{k}=v_{k-1} u_{k}-u_{k-1} v_{k}, \quad \hat{\omega}_{k}=\phi_{k-1} \theta_{k}-\theta_{k-1} \phi_{k}, \\
& r_{j k}=r\left(t_{j}, t_{k}\right), \quad \rho_{j k}=\rho\left(t_{j}, t_{k}\right),
\end{aligned}
$$

$R$ is the $m x m$ matrix with elements $r_{j k} ; j, k=1,2, \cdots, m$, $P$ is the $m x m$ matrix with elements $\rho_{g k} ; j, k=1,2, \cdots, m$,

$|R|$ and $|P|$ are the determinants of $R$ and $P$ respectively,

$$
\begin{aligned}
\bar{x} & =\left(x_{1}, x_{2}, \cdots, x_{m}\right), \\
\bar{y} & =\left(y_{1}, y_{2}, \cdots, y_{m}\right), \\
\overline{\Delta p} & =\left(p_{1}-p_{0}, p_{2}-p_{1}, p_{m}-p_{m-1}\right) .
\end{aligned}
$$

\section{Some lemmas.}

Lemma 1. Under (A), (B) and (D) of Definition 1,

(a) $u(t)>0, \theta(t)>0$ for $t \in(a, b]$,

(b) $\hat{w}_{k}>0, \hat{\omega}_{k}>0$ for $k=1,2, \cdots, m ; n>0$,

(c) $R^{-1}$ and $P^{-1}$ exist.

Proof. $\quad d / d t[u(t) / v(t)]=w(t) / v^{2}(t)>0$ by (B) and (D). Hence $u(t) / v(t)$ increases as $t$ increases and so $u(t)>v(t) u(a) / v(a), t \in(a, b]$, and $0<$ $\left[u_{k} / v_{k}\right]-\left[u_{k-1} / v_{k-1}\right]=\widehat{w}_{k} /\left[v_{k} v_{k-1}\right], \quad k=1,2, \cdots, m$, giving the first parts of (a) and (b). One may actually compute $R^{-1}$, the result being 


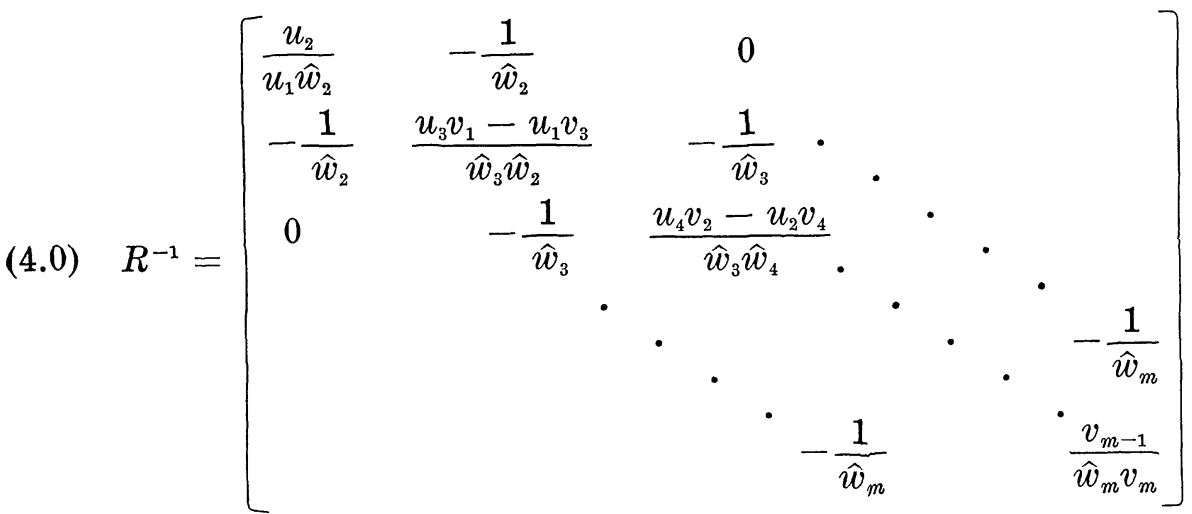

We remark for later reference that $|R|$ may also be computed explicitly.

$$
|R|=u_{1} \hat{w}_{2} \hat{w}_{3} \cdots \hat{\omega}_{m} v_{m} .
$$

Completely analogous results hold for $\theta(t), \hat{w}_{k}, P^{-1}$, and $|P|$.

Lemma 2. Under (A)-(D) of Definition 1,

(a) $2^{n} \widehat{w}_{k}, 2^{n} \hat{\omega}_{k}$ are bounded uniformly (in $k$ and $n$ ) away from 0 ,

(b) if $w(t) \equiv \omega(t)$, then $\lim _{n \rightarrow \infty}\left|\hat{\omega}_{k}-\widehat{w}_{k}\right| 2^{2 n}=0$ uniformly (in $k$ ).

Proof. A simple algebraic manipulation followed by use of the mean value the theorem for derivatives gives

$$
2^{n} \widehat{w}_{k}=w\left(t_{k-1}\right)+2^{-n-1}[b-a]\left[v\left(t_{k-1}\right) u^{\prime \prime}\left(X_{1 k}\right)-u\left(t_{k-1}\right) v^{\prime \prime}\left(X_{2 k}\right)\right]
$$

where $t_{k-1}<X_{1 k}, X_{2 k}<t_{k}$. That $2^{n} \hat{w}_{k}$ is uniformly bounded away from 0 now follows from the fact that $w$ is (uniformly) continuous and positive on $[a, b]$ and hence bounded away from 0 and from the fact that the second term in the expression for $2^{n} \hat{w}_{k}$ becomes uniformly small as $n$ gets large. A similar argument is pertinent for $2^{n} \hat{\omega}_{k}$ giving us part (a).

A somewhat more lengthy algebraic exercise together with application of Taylor's formula (two terms plus remainder) gives

$$
\begin{aligned}
& 2^{2 n}\left[\hat{\omega}_{k}-\hat{w}_{k}\right] \\
&= 2^{2 n}\left\{\left[\omega\left(t_{k-1}\right)-w\left(t_{k-1}\right)\right][b-a] 2^{-n}+\left[\omega^{\prime}\left(t_{k-1}\right)-w^{\prime}\left(t_{k-1}\right)\right][b-a]^{2} 2^{-2 n-1}\right\} \\
&+\left[(b-a)^{2} / 2\right]\left\{\phi\left(t_{k-1}\right)\left[\theta^{\prime \prime}\left(X_{1 k}\right)-\theta^{\prime \prime}\left(t_{k-1}\right)\right]-\theta\left(t_{k-1}\right)\left[\phi^{\prime \prime}\left(X_{2 k}\right)-\phi^{\prime \prime}\left(t_{k-1}\right)\right]\right. \\
&\left.-v\left(t_{k-1}\right)\left[u^{\prime \prime}\left(X_{3 k}\right)-u^{\prime \prime}\left(t_{k-1}\right)\right]-u\left(t_{k-1}\right)\left[v^{\prime \prime}\left(X_{4 k}\right)-v^{\prime \prime}\left(t_{k-1}\right)\right]\right\} .
\end{aligned}
$$

Since $\omega(t) \equiv w(t)$ (and hence $\omega^{\prime}(t) \equiv w(t)$ ) and since $\theta^{\prime \prime}, \phi^{\prime \prime}, u^{\prime \prime}$ and $v^{\prime \prime}$ are (uniformly) continuous on $[a, b]$, it follows that $(b)$ holds.

Lemma 3. If Conditions (A)-(F) of Definition 1 and Theorem 1 hold, then 
(a) $\lim _{n \rightarrow \infty}[|R| /|P|]=C_{1}$,

(b) $\lim _{n \rightarrow \infty} x_{1}^{2}\left\{\frac{1}{u_{1} v_{1}}-\frac{1}{\theta_{1} \phi_{1}}\right\}=C_{2} x^{2}$ (a) for almost all $x$ ( $m_{r}$ sense),

(see Theorem 1 for the definitions of $C_{1}$ and $C_{2}$ ).

Proof. Using formula (4.1) for $|R|$ and its analog for $|P|$ we have

$$
\ln [|R| /|P|]=\ln \left[u_{1} \mid \theta_{1}\right]+\ln \left[v_{m} / \phi_{m}\right]+\sum_{k=2}^{m} \ln \left[\hat{w}_{k} / \hat{\omega}_{k}\right] .
$$

Now

$$
\left|\ln \left[\hat{w}_{k} / \hat{\omega}_{k}\right]\right|=\left|\ln \hat{w}_{k}-\ln \hat{\omega}_{k}\right|=\left|\hat{w}_{k}-\hat{\omega}_{k}\right| / X_{k}
$$

where $\min \left(\hat{\omega}_{k}, \hat{w}_{k}\right) \leqq X_{k} \leqq \max \left(\hat{\omega}_{k}, \hat{w}_{k}\right)$. But by Lemma $2,\left|\hat{w}_{k}-\hat{\omega}_{k}\right|=$ $o\left(2^{-2 n}\right)$ uniformly in $k$ while $X_{k} 2^{n}$ is bounded away from 0 uniformly in $k$ and $n$. It follows that

$$
\lim _{n \rightarrow \infty} \sum_{k=2}^{m} \ln \left[\hat{w}_{k} / \hat{\omega}_{k}\right]=0 .
$$

Hence

$$
\lim _{n \rightarrow \infty} \ln [|R| /|P|]=\lim _{n \rightarrow \infty} \ln \left[u_{1} / \theta_{1}\right]+\ln [v(b) / \phi(b)] .
$$

This gives part (a) immediately in ca: $\theta(a) \neq 0$. If $\theta(a)=0$ (and hence $u(a)=0)$ we use the fact that $w(t) \equiv \omega(t)$ to write

$$
v^{2}(t) \frac{d}{d t}\left\{\frac{u(t)}{v(t)}\right\} \equiv \phi^{2}(t) \frac{d}{d t}\left\{\frac{\theta(t)}{\phi(t)}\right\}
$$

so that

$$
\int_{a}^{t} d[u(s) / v(s)] \equiv \int_{a}^{t}\left[\phi^{2}(s) / v^{2}(s)\right] d[\theta(s) / \phi(s)] .
$$

By the mean value theorem for integrals and the fact that $u(\alpha)=\theta(a)=0$, we have

$$
\left.u(t) / v(t)=\left[\phi^{2}(X) \theta(t)\right] / v^{2}(X) \phi(t)\right] \text { for some } X, a<X<t .
$$

Rewriting and letting $t \rightarrow 0$, we see that $\lim _{t \rightarrow 0}[u(t) / \theta(t)]=\phi(\alpha) / v(\alpha)$, the needed result.

Part (b) is immediate in case $\theta(a)$ and $u(a)$ are not zero. If $\theta(a)=$ $u(a)=0$, then $x(a)=0$ with $m_{r}$ (and $m_{\rho}$ ) measure one. Hence it will be sufficient to show that $\left[1 / u_{1} v_{1}\right]-\left[1 / \theta_{1} \phi_{1}\right]$ is bounded. But $\left[1 / u_{1} v_{1}\right]-$ $\left[1 / \theta_{1} \phi_{1}\right]=\left\{\left[\phi_{1} / u_{1}\right]-\left[v_{1} / \theta_{1}\right]\right\} /\left[v_{1} \phi_{1}\right]$ which will be bounded if $\left[\phi_{1} / u_{1}\right]-\left[v_{1} / \theta_{1}\right]$ is bounded. Now 


$$
\begin{aligned}
& \left|\frac{\phi(t)}{u(t)}-\frac{v(t)}{\theta(t)}\right|=\frac{|\phi(t) \theta(t)-u(t) v(t)|}{u(t) \theta(t)} \\
& =\frac{\left|\phi(t) \theta(t)-\left[\phi^{2}(X) \theta(t) v^{2}(t)\right] /\left[v^{2}(X) \phi(t)\right]\right|}{u(t) \theta(t)}
\end{aligned}
$$

where $a<X<t$, (by 4.2),

$$
=\frac{\left|\phi^{2}(t) v^{2}(X)-\phi^{2}(X) v^{2}(t)\right|}{u(t) v^{2}(X) \phi(t)}
$$$$
=\frac{\left|v^{2}(X) \frac{\phi^{2}(t)-\phi^{2}(\alpha)}{t-a}+\phi^{2}(a) \frac{v^{2}(X)-v^{2}(t)}{t-a}+v^{2}(t) \frac{\phi^{2}(\alpha)-\phi^{2}(X)}{t-a}\right|}{v^{2}(X) \phi(t) v(t) \frac{[u(t) / v(t)]-[u(\alpha) / v(\alpha)]}{t-a}}
$$

$$
=\frac{\left|2 v^{2}(X) \phi^{\prime}\left(X_{1}\right) \phi\left(X_{1}\right)+2 \phi^{2}(a) v^{\prime}\left(X_{2}\right) v\left(X_{2}\right) \frac{t-X}{t-a}+2 v^{2}(t) \phi^{\prime}\left(X_{3}\right) \phi\left(X_{3}\right) \frac{X-a}{t-a}\right|}{v^{2}(X) \phi(t) v(t) w\left(X_{4}\right) / v^{2}\left(X_{4}\right)}
$$

where $a<X_{1}<t, X<X_{2}<t, a<X_{3}<X$, $a<X_{4}<t$. This last] expression is clearly uniformly bounded for $t \in(a, b]$.

The first three lemmas allow us to prove the following key result.

LEMmA 4. If (A)-(F) of Definition 1 and Theorem 1 hold, then

$$
\lim _{n \rightarrow \infty} \bar{x}^{\prime}\left(R^{-1}-P^{-1}\right) \bar{x}=C_{2} x^{2}(a)+\int_{a}^{b} f(t) d\left\{x^{2}(t) /[\phi(t) v(t)]\right\}
$$

Proof. We may verify using formula (4.0) for $R^{-1}$ and its analog for $P^{-1}$ that

$$
\begin{aligned}
\bar{x}^{\prime}\left(R^{-1}-P^{-1}\right) \bar{x} & =\frac{x_{1}^{2}}{u_{1} v_{1}}-\frac{x_{1}^{2}}{\theta_{1} \phi_{1}}+\sum_{k=2}^{m}\left\{\frac{\left(v_{k-1} x_{k}-v_{k} x_{k-1}\right)^{2}}{v_{k-1} v_{k} \widehat{w}_{k}}-\frac{\left(\phi_{k-1} x_{k}-\phi_{k} x_{k-1}\right)^{2 !}}{\phi_{k-1} \phi_{k} \widehat{w}_{k}}\right\} \\
& =J_{n}(x)+K_{n}(x)+L_{n}(x)
\end{aligned}
$$

where

$$
\begin{aligned}
& J_{n}(x)=x_{1}^{2}\left\{\frac{1}{u_{1} v_{1}}-\frac{1}{\theta_{1} \phi_{1}}\right\}, \\
& K_{n}(x)=\sum_{k=2}^{m}\left[\hat{\omega}_{k}-\hat{w}_{k}\right]\left[\phi_{k-1} x_{k}-\phi_{k} x_{k-1}\left[^{2} /\left[\phi_{k-1} \phi_{k} \hat{\omega}_{k} \hat{w}_{k}\right],\right.\right. \\
& L_{n}(x)=\sum_{k=2}^{m} \hat{\omega}_{k}\left\{\frac{\phi_{k-1} \phi_{k}\left[v_{k-1} x_{k}-v_{k} x_{k-1}\right]^{2}-v_{k-1} v_{k}\left[\phi_{k-1} x_{k}-\phi_{k} x_{k-1}\right]^{2}}{v_{k-1} v_{k} \phi_{k-1} \phi_{k} \hat{\omega}_{k} \hat{w}_{k}}\right\} .
\end{aligned}
$$

We note first that $J_{n}(x) \rightarrow C_{2} x^{2}(a)$ as $n \rightarrow \infty$ by Lemma 3 part (b). We show next that $K_{n}(x) \rightarrow 0$ as $n \rightarrow \infty$. Let $\varepsilon>0$ be given and choose $N$ so large that $n \geqq N$ implies that $m^{2}\left|\hat{\omega}_{k}-\widehat{w}_{k}\right|<\varepsilon$, (see Lemma 2). Let $\tau=\min _{0 \leqq k \leqq m, n>0}\left[m^{2} \phi_{k-1} \phi_{k} \hat{\omega}_{k} \hat{w}_{k}\right]$. Then for $n \geqq N$, 


$$
\begin{aligned}
\left|K_{n}\right| & =\left|\sum_{k=2}^{m} \frac{m^{2}\left(\hat{\omega}_{k}-\hat{w}_{k}\right)}{m^{2} \phi_{k-1} \phi_{k} \hat{\omega}_{k} \hat{w}_{k}}\left\{x_{k} \frac{\phi_{k-1}-\phi_{k}}{t_{k}-t_{k-1}}+\phi_{k} \frac{x_{k}-x_{k-1}}{t_{k}-t_{k-1}}\right\}\left(t_{k}-t_{k-1}\right)^{2}\right| \\
& \leqq(\varepsilon / \tau) \sum_{k=2}^{m}\left\{-x\left(t_{k}\right) \phi^{\prime}\left(X_{k}\right)+\phi\left(t_{k}\right) \frac{x\left(t_{k}\right)-x\left(t_{k-1}\right)}{t_{k}-t_{k-1}}\right\}^{2}\left(t_{k}-t_{k-1}\right)^{2}
\end{aligned}
$$

where $t_{k-1}<X_{k}<t_{k}$. Thus

$$
\begin{aligned}
\left|K_{n}\right| \leqq & {[\varepsilon(b-a) / \tau m] \sum_{k=2}^{m}\left[x\left(t_{k}\right) \phi^{\prime}\left(X_{k}\right)\right]^{2}\left[t_{k}-t_{k-1}\right] } \\
& +(2 \varepsilon / \tau)\left|\sum_{k=2}^{m} x^{2}\left(t_{k}\right) \phi\left(t_{k}\right) \phi^{\prime}\left(t_{k}\right)\left(t_{k}-t_{k-1}\right)\right| \\
& +(2 \varepsilon / \tau)\left|\sum_{k=2}^{m} x\left(t_{k}\right) x\left(t_{k-1}\right) \phi\left(t_{k}\right) \phi^{\prime}\left(X_{k}\right)\left(t_{k}-t_{k-1}\right)\right| \\
& +(\varepsilon / \tau) \max _{a \leqq t \leqq b} \phi^{2}(t) \sum_{k=2}^{m}\left[x(t)-x\left(t_{k-1}\right)\right]^{2} .
\end{aligned}
$$

The first three terms are small since the sums involved are Riemann sums. Futhermore the sum in the fourth term approaches a limit by Baxter's theorem. The result now follows.

Lastly, we consider $L_{n}(x)$.

$$
\begin{aligned}
& L_{n}(x) \\
& =\sum_{k=2}^{m} \frac{\phi_{k-1} \phi_{k}\left[v_{k-1} x_{k}-v_{k} x_{k-1}\right]^{2}-v_{k-1} v_{k}\left[\phi_{k-1} x_{k}-\phi_{k} x_{k-1}\right]^{2}}{v_{k-1} v_{k} \phi_{k-1} \phi_{k} \hat{w}_{k}} \\
& =\sum_{k=1}^{m}\left\{\frac{\phi_{k} v_{k-1}-\phi_{k-1} v_{k}}{\hat{w}_{k}}\right\}\left\{\frac{\phi_{k-1} v_{k-1} x_{k}^{2}-x_{k-1}^{2} v_{k} \phi_{k}}{v_{k-1} v_{k} \phi_{k-1} \phi_{k}}\right\} \\
& =\sum_{k=2}^{m}\left\{\frac{v_{k}\left(\phi_{k}-\phi_{k-1}\right)-\phi_{k}\left(v_{k}-v_{k-1}\right)}{v_{k}\left(u_{k}-u_{k-1}\right)-u_{k}\left(v_{k}-v_{k-1}\right)}\right\}\left\{\frac{x_{k}^{2}}{\phi_{k} v_{k}}-\frac{x_{k-1}^{2}}{\phi_{k-1} v_{k-1}}\right\} \\
& =\sum_{k=2}^{m}\left\{\frac{v_{k} \phi_{k}^{\prime}-\phi_{k} v_{k}^{\prime}+\left[v_{k} \phi^{\prime \prime}\left(X_{1 k}\right)-\phi_{k} v^{\prime \prime}\left(X_{2 k}\right)\right][(b-a) / 2 m]}{v_{k} u_{k}^{\prime}-u_{k} v_{k}^{\prime}+\left[v_{k} u^{\prime \prime}\left(X_{3 k}\right)-u_{k} v^{\prime \prime}\left(X_{4 k}\right)\right][(b-a) / 2 m]}\right\}\left\{\frac{x_{k}^{2}}{\phi_{k} v_{k}}-\frac{x_{k-1}^{2}}{\phi_{k-1} v_{k-1}}\right\}
\end{aligned}
$$

(by Taylor's formula)

$$
=\sum_{k=2}^{m}\left\{\frac{v\left(t_{k}\right) \phi^{\prime}\left(t_{k}\right)-\phi\left(t_{k}\right) v^{\prime}\left(t_{k}\right)}{v\left(t_{k}\right) u^{\prime}\left(t_{k}\right)-u\left(t_{k}\right) v^{\prime}\left(t_{k}\right)}+B_{k m} / m\right\}\left\{\frac{x^{2}\left(t_{k}\right)}{\phi\left(t_{k}\right) v\left(t_{k}\right)}-\frac{x^{2}\left(t_{k-1}\right)}{\phi\left(t_{k-1}\right) v\left(t_{k-1}\right)}\right\}
$$

(where $B_{k m}$ is bounded independently of $k$ and $m$ )

$$
\rightarrow \int_{\alpha}^{\beta} f(t) d\left\{x^{2}(t) /[\phi(t) v(t)]\right\} \quad \text { as } n \rightarrow \infty .
$$

For later re rence we note that the last expression for $L_{n}(x)$ may be rewritten using partial summation giving

$$
\text { (4.4) } \begin{aligned}
L_{n}(x)= & \left\{\frac{x^{2}\left(t_{m}\right)}{\phi\left(t_{m}\right) v\left(t_{m}\right)}\right\}\left\{f\left(t_{m}\right)+O(1 / m)\right\}-\left\{\frac{x^{2}\left(t_{1}\right)}{\phi\left(t_{1}\right) v\left(t_{1}\right)}\right\}\left\{f\left(t_{2}\right)+O(1 / m)\right\} \\
& -\sum_{k=2}^{m-1} \frac{x^{2}\left(t_{k}\right)}{\phi\left(t_{k}\right) v\left(t_{k}\right)}\left[f\left(t_{k+1}\right)-f\left(t_{k}\right)\right] .
\end{aligned}
$$




\section{Proof of Theorem 1.}

Necessity. Here we assume that $m_{\rho}$ is equivalent to $m_{r}$. Now by the corollary to Baxter's theorem, $f_{\rho}(t) \equiv f_{r}(t)$. An easy calculation shows that $f_{r}(t) \equiv v(t) u^{\prime}(t)-u(t) v^{\prime}(t)$ and $f_{\rho}(t) \equiv \phi(t) \theta^{\prime}(t)-\theta(t) \phi^{\prime}(t)$ so that Condition (E) holds. To see Condition $(\mathrm{F})$, we note that $x(a)=0$ with $m_{\rho}$ measure 0 or 1 according as $\theta(a) \neq 0$ or $\theta(a)=0$. Similarly $x(a)=0$ with $m_{r}$ measure 0 or 1 according as $u(a) \neq 0$ or $u(a)=0$. But since $m_{\rho}$ is equivalent to $m_{r}$, null sets with respect to the two measures must correspond. Hence $F$ holds.

Sufficiency. We assume that Conditions (E) and (F) of the theorem are satisfied. Define two functions $F_{M}$ and $F_{M, n}$ on $C[a, b]$ by

$$
\begin{aligned}
& F_{M}(y)=\left\{\begin{array}{ll}
1 & \text { if } \sup _{a \leqq t \leqq b}|y(t)| \leqq M \\
0 & \text { otherwise }
\end{array}\right\} \\
& F_{M, n}(y)=\left\{\begin{array}{ll}
1 & \text { if } \sup _{1 \leqq k \leqq m}\left|y\left(t_{k}\right)\right| \leqq M \text { and } \sum_{k=2}^{m}\left[y\left(t_{k}\right)-y\left(t_{k-1}\right)\right]^{2} \leqq \int_{a}^{b} w(t) d t+1 \\
0 & \text { otherwise }
\end{array}\right\} .
\end{aligned}
$$

Let $H_{M, n}$ be a function of $m$ real variables $\bar{u}=\left(u_{1}, u_{1}, \cdots, u_{m}\right)$ such that

$$
H_{M, n}(\bar{u})=\left\{\begin{array}{ll}
1 & \text { if } \left.\sup _{1 \leqq k \leqq m}\left|u_{k}\right| \leqq M \text { and } \sum_{k=2}^{m}\left[u_{k}-u_{k-1}\right]^{2} \leqq \int_{a}^{b} w(t) d t+1\right\} \\
0 & \text { otherwise }
\end{array}\right\}
$$

and note that $H_{M, n}(\bar{y})=F_{M, n}(y)$. By Baxter's theorem and using the continuity of $y$ and the fact that $f_{p}(t) \equiv f_{r}(t) \equiv w(t)$, we have that for almost all $y \in C$ (in the sense of both measures $m_{\rho}$ and $\left.m_{r}\right) \lim _{n \rightarrow \infty} F_{M, n}(y)=$ $F_{M}(y)$ and $\lim _{M \rightarrow \infty} F_{M}(y)=1$. Hence for any step function $p$ on $[a, b]$,

$$
\begin{aligned}
& E^{\rho}\left\{F_{M}(y) \exp \int_{\alpha}^{\beta} y(t) d p(t)\right\}=F^{\rho}\left\{\lim _{n \rightarrow \infty}\left[F_{M, n}(y) \exp \sum_{k=1}^{m} y\left(t_{k}\right)\left[p\left(t_{k}\right)-p\left(t_{k-1}\right)\right]\right]\right\} \\
&\left.=E^{\rho}\left\{\lim _{n \rightarrow \infty}\left[H_{M, n}(\bar{y}) \exp \bar{y}^{\prime} \overline{\Delta p}\right)\right]\right\} \\
&=\lim _{n \rightarrow \infty} E^{\rho}\left\{H_{M, n}(\bar{y}) \exp \left(\bar{y}^{\prime} \overline{\Delta p}\right)\right\} \quad \text { (by bounded convergence) } \\
&=\lim _{n \rightarrow \infty}\left[(2 \pi)^{m}|P|\right]^{-1 / 2} \int_{-\infty}^{\infty} H_{M, n}(\bar{u}) \exp \left[\bar{u}^{\prime} \overline{\Delta p}-(1 / 2) \bar{u}^{\prime} P^{-1} \bar{u}\right] \overline{d u} \\
&=\lim _{n \rightarrow \infty}\left\{[|P| /|R|]\left[(2 \pi)^{m}|R|\right]\right\}^{-1 / 2} \int_{a}^{b} H_{M, n}(\bar{u}) \\
& \times \exp \left\{\bar{u}^{\prime} \overline{\Delta p}+(1 / 2)\left[\bar{u}^{\prime}\left(R^{-1}-P^{-1}\right) \bar{u}-\bar{u}^{\prime} R^{-1} \bar{u}\right]\right\} \overline{d u} \\
&= \lim _{n \rightarrow \infty}[|R| /|P|]^{1 / 2} \lim _{n \rightarrow \infty} E^{r}\left\{H_{M, n}(\bar{x}) \exp \left[\bar{x}^{\prime} \overline{\Delta p}+(1 / 2) \bar{x}^{\prime}\left(R^{-1}-P^{-1}\right) \bar{x}\right]\right\} \\
&= C_{1} \lim _{n \rightarrow \infty} E^{r}\left\{F_{M, n}(x) \exp \left[\bar{x}^{\prime} \overline{\Delta p}+(1 / 2) \bar{x}^{\prime}\left(R^{-1}-P^{-1}\right) \bar{x}\right]\right\},
\end{aligned}
$$


the last equality following from Lemma 3, part (a). Now the expectant will be bounded (independently of $n$ and $x$ ) provided $\bar{x}^{\prime} \overline{\Delta p}+(1 / 2) \bar{x}^{\prime}\left(R^{-1}-P^{-1}\right) \bar{x}$ is so bounded on the subset $A_{n}$ of $C$ where $F_{M, n}$ is different from 0 . But on $A_{n}, \overline{x^{\prime}} \overline{\Delta p} \leqq M \sum_{k=1}^{m}\left|p\left(t_{k}\right)-p\left(t_{k-1}\right)\right|$ which is bounded (independently of $n$ and $x)$. Furthermore, $\bar{x}^{\prime}\left(R^{-1}-P^{-1}\right) \bar{x}=J_{n}(x)+K_{n}(x)+L_{n}(x) . J_{n}(x)$ is bounded on $A_{n}$ as may be seen from the proof of Lemma 3 , part (b), while $K_{n}(x)$ and $L_{n}(x)$ may been to be bounded on $A_{n}$ by examination of formulas (4.3) and (4.4) respectively. This allows us to take the limit inside the expected value from which we obtain (see Lemma 4)

$$
\begin{aligned}
& E^{\rho}\left\{F_{M}(y) \exp \int_{a}^{b} y(t) d p(t)\right\} \\
& =C_{1} E^{r}\left\{F_{M}(x) \exp \left[\int_{a}^{b} x(t) d p(t)+(1 / 2)\left(C_{2} x^{2}(a)+\int_{a}^{b} f(t) d\left\{x^{2}(t) /[\phi(t) v(t)]\right\}\right]\right\} .\right.
\end{aligned}
$$

Now letting $M \rightarrow \infty$, we obtain by monotone convergence

$$
\begin{aligned}
& E^{\mathrm{\rho}}\left\{\exp \int_{a}^{b} y(t) d p(t)\right\} \\
& \quad=C_{1} E^{r}\left\{\exp \left[\int_{a}^{b} x(t) d p(t)+(1 / 2)\left(C_{2} x^{2}(a)+\int_{a}^{b} f(t) d\left\{x^{2}(t) /[\phi(t) v(t)]\right\}\right]\right\} .\right.
\end{aligned}
$$

Now consider the stochastic process $\{z(t), a \leqq t \leqq b\}$ with space of sample functions $\{C, B\}$ whose Radon-Nikodym derivative with respect to $\{x(t), a \leqq t \leqq b\}$ is $C_{1} \exp [1 / 2]\left[C_{2} x^{2}(a)+\int_{a}^{b} f(t) d\left\{x^{2}(t) /[\phi(t) v(t)]\right\}\right.$. Then for all measurable (B) functions $F$,

$$
E\{F(z)\}=C_{1} E^{r}\left\{F(x) \exp [1 / 2]\left[C_{2} x^{2}(\alpha)+\int_{a}^{b} f(t) d\left\{x^{2}(t) /[\phi(t) v(t)]\right\}\right]\right\} .
$$

Hence in particular, formula (4.6) holds for the process $\{z(t), a \leqq t \leqq b\}$. But this means that $\{z(t), a \leqq t \leqq b\}$ and $\{y(t), a \leqq t \leqq b\}$ have the same multidimensional moment generating functions and since they determine the measures of all the measurable subsets of $C$ (i.e., all sets in B), the processes $z(t)$ and $y(t)$ are identical. Since (4.7) holds for the former, it also holds for the latter. This shows that $m_{\rho}$ is absolutely continuous with respect to $m_{r}$. By symmetry, $m_{r}$ is also absolutely continuous with respect to $m_{\rho}$. This completes the proof.

5. Examples. The best known process in the class $M[0, T]$ is the Wiener process (Brownian motion process) with probability measure $m_{w_{\sigma}}$ determined by the covariance function

$$
w_{\sigma}(s, t)=\sigma^{2} \min (s, t)=\left\{\begin{array}{ll}
\sigma^{2} s & s \leqq t \\
\sigma^{2} t & s \geqq t
\end{array}\right\}, \quad \sigma^{2}>0 .
$$


Theorem 1 shows immediately that two such processes determined by covariance functions $w_{\sigma_{1}}$ and $w_{\sigma_{2}}$ are equivalent if and only if $\sigma_{1}=\sigma_{2}$, (see [2] for a discussion of what is essentially this problem). We can actually say much more. In fact, we can easily characterize all processes in the class $M[0, T]$ which are equivalent to the Wiener process determined by $w_{\sigma}(s, t)^{6}$. Let $\phi$ be any function which is positive and has a continuous second derivative on $[0, T]$ and define $\theta$ by $\theta(s)=$ $\sigma^{2} \phi(s) \int_{0}^{s}\left[1 / \phi^{2}(t)\right] d t$. Then those processes (and only those) in the class $M[0, T]$ with covariance functions of the type

$$
\rho(s, t)=\left\{\begin{array}{ll}
\theta(s) \phi(t) & s \leqq t \\
\theta(t) \phi(s) & s \geqq t
\end{array}\right\}
$$

are equivalent to the process in $M[0, T]$ determined by $w_{\sigma}(s, t)$. We give two examples.

EXAMPLE 1. Let $\{y(t), 0 \leqq t \leqq T<1\}$ be the process belonging to $M[0, T]$ with probability measure $m_{\rho}$ determined by the covariance function

$$
\rho(s, t)=\left\{\begin{array}{ll}
s(1-t) & s \leqq t \\
t(1-s) & s \geqq t
\end{array}\right\}^{7} .
$$

'This process is equivalent to the Wiener process $\{x(t), 0 \leqq t \leqq T\}$ with covariance function $w_{1}(s, t)=\min (s, t)$. Moreover,

$$
d m_{\rho} / d m_{w_{1}}=(1-T)^{-1 / 2} \exp \left\{-x^{2}(T) /[2(1-T)]\right\} .
$$

EXAMPLE 2. Let $\{y(t), 0 \leqq t \leqq 1\}$ be the process belonging to $M[0,1]$ with probability measure $m_{\rho_{\lambda}}$ determined by the covariance function

$$
\rho_{\lambda}(s, t)=\left\{\begin{array}{ll}
\frac{\sin \sqrt{\lambda} s \cos \sqrt{\lambda}(1-t)}{\sqrt{\bar{\lambda}} \cos \sqrt{\bar{\lambda}}} & s \leqq t \\
\frac{\sin \sqrt{\bar{\lambda}} t \cos \sqrt{\lambda}(1-s)}{\sqrt{\bar{\lambda} \cos \sqrt{\bar{\lambda}}}} & s \leqq t
\end{array}\right\}^{8} \quad \lambda<\left(\pi^{2} / 4\right) .
$$

This process is equivalent to the Wiener process $\{x(t), 0 \leqq t \leqq 1\}$ with covariance function $w_{1}(s, t)=\min (s, t)$. Moreover,

$$
d m_{\rho_{\lambda}} / d m_{w_{1}}=(\cos \sqrt{\lambda})^{1 / 2} \exp \left[(\lambda / 2) \int_{0}^{1} x^{2}(t) d t\right] .
$$

${ }^{6} \mathrm{We}$ reason as follows. For $r(s, t)=w_{\sigma}(s, t), w(s)=\sigma^{2}$. Hence $\omega(s)$ must equal $\sigma^{2}$, i.e., $d / d s[\theta(s) / \phi(s)]=\sigma^{2} / \phi^{2}(s)$. This together with $\theta(0)=0$ implies that $\theta(s)=\sigma^{2} \phi(s) \int_{0}^{s}\left[1 / \phi^{2}(t)\right] d t$.

7 This process has been studied by various authors, among them Doob [4].

8 For $\lambda=0, \rho_{\lambda}(s, t)=\lim _{\lambda \rightarrow 0} \rho_{\lambda}(s, t)=\min (s, t)$. 
For our last example we consider the class of (Ornstien Uhlenbeck) processes in $M[a, b]$ determined by covariance functions of the type

$$
\rho_{\sigma, \beta}(s, t)=\sigma^{2} \exp [-\beta|s-t|]=\left\{\begin{array}{ll}
\theta(s) \phi(t) & s \leqq t \\
\theta(t) \phi(s) & s \geqq t
\end{array}\right\}
$$

where $\theta(t)=\sigma^{2} \exp (\beta t), \phi(t)=\exp (-\beta t), \quad\left(\sigma^{2}>0, \beta>0\right)$. If $U[a, b\rceil$ denotes the class of such processes, we have the following result.

ExAMPLE 3. Let $\{x(t), 0 \leqq t \leqq T\}$ and $\{y(t), 0 \leqq t \leqq T\}$ be two processes belonging to $U[0, T]$ with covariance functions $\rho_{\sigma_{0}, \beta_{0}}$ and $\rho_{\sigma_{1}, \beta 2}$ respectively determining two probability measures $m_{\sigma_{0}, \beta_{0}}$ and $m_{\sigma_{1}, \beta_{1}}$ on $\{C, B\}$. Then $m_{\sigma_{0}, \beta_{0}}$ is equivalent to $m_{\sigma_{1}, \beta_{1}}$ if and only if $\sigma_{0}^{2} \beta_{0}=\sigma_{1}^{2} \beta_{1}$. Moreover if this condition is satisfied and if we let $K=2 \sigma_{0}^{2} \beta_{0}=2 \sigma_{1}^{2} \beta_{1}$, then

$$
\begin{aligned}
& \frac{d m_{\sigma_{1}, \beta_{1}}}{d m_{\sigma_{0}, \beta_{0}}}=\left(\beta_{1} / \beta_{0}\right)^{1 / 2} \\
& \quad \times \exp \left\{(-1 / 2 K)\left[\left(\beta_{1}-\beta_{0}\right)\left(x^{2}(0)+x^{2}(T)-K T\right)+\left(\beta_{1}^{2}-\beta_{0}^{2}\right) \int_{0}^{T} x^{2}(t) d t\right]\right\}^{9} .
\end{aligned}
$$

6. Conjecture. Consider two general Gaussian processes determined by covariance functions $r(s, t)$ and $\rho(s, t)$ respectively. Under regularity and boundary conditions of the type (A)-(D) of Definition 1, a necessary and sufficient condition that the two processes be equivalent is that $f_{r}(t) \equiv f_{\mathrm{\rho}}(t)$, (see $\left.\S 2\right)$.

\section{REFERENCES}

1. Glen Baxter, A strong limit theorem for Gaussian processes, Proc. Amer. Math. Soc., 7 (1956), 522-527.

2. R. H. Cameron and W. T. Martin, The Behavior of measure and measurability under change of scale in Wiener space, Bull. Amer. Math. Soc., 53 (1947), 130-137.

3. J. L. Doob, Stochastic Processes, New York, 1953.

4. - Heuristic approach to the Kolmogorov-Smirnov theorems, Ann. of Math. Stat., 20 (1949), 393-403.

5. Jacob Feldman, Equivalence and perpendicularity of Gaussian processes, Pacific J. Math., 8 (1958), 699-708.

6. I. E. Segal, Distributions in Hilbert space and canonical systems of operators, Trans. Amer. Math. Soc., 88 (1958), 12-41.

7. Charlotte T. Striebel, Densities for stochastic processes, Ann. Math. Stat., 30 (1959), 559-567.

8. Dale E. Varberg, Some Radon-Nikodym Derivatives Associated with Stochastic Processes, Thesis, 1959, University of Minnesota.

HAMLINE UNIVERSITY

9 This result is due to Charlotte T. Striebel, the formula above being displayed near the top of page 566 in [7]. 


\title{
PACIFIC JOURNAL OF MATHEMATICS
}

\author{
EDITORS
}

\author{
Ralph S. Phillips \\ Stanford University \\ Stanford, California \\ F. H. Brownell \\ University of Washington \\ Seattle 5, Washington
}

\author{
A. L. Whiteman \\ University of Southern California \\ Los Angeles 7, California \\ L. J. PAige \\ University of California \\ Los Angeles 24, California
}

ASSOCIATE EDITORS
E. F. BECKENBACH
D. DERRY
H. L. ROYDEN
E. G. STRAUS
T. M. CHERRY
M. OHTSUKA
E. SPANIER
F. WOLF

\section{SUPPORTING INSTITUTIONS}

\author{
UNIVERSITY OF BRITISH COLUMBIA \\ CALIFORNIA INSTITUTE OF TECHNOLOGY \\ UNIVERSITY OF CALIFORNIA \\ MONTANA STATE UNIVERSITY \\ UNIVERSITY OF NEVADA \\ NEW MEXICO STATE UNIVERSITY \\ OREGON STATE COLLEGE \\ UNIVERSITY OF OREGON \\ OSAKA UNIVERSITY \\ UNIVERSITY OF SOUTHERN CALIFORNIA
}

\author{
STANFORD UNIVERSITY \\ UNIVERSITY OF TOKYO \\ UNIVERSITY OF UTAH \\ WASHINGTON STATE COLLEGE \\ UNIVERSITY OF WASHINGTON \\ * * * * \\ AMERICAN MATHEMATICAL SOCIETY \\ CALIFORNIA RESEARCH CORPORATION \\ HUGHES AIRCRAFT COMPANY \\ SPACE TECHNOLOGY LABORATORIES \\ NAVAL ORDNANCE TEST STATION
}

Mathematical papers intended for publication in the Pacific Journal of Mathematics should be typewritten (double spaced), and the author should keep a complete copy. Manuscripts may be sent to any one of the four editors. All other communications to the editors should be addressed to the managing editor, L. J. Paige at the University of California, Los Angeles 24, California.

50 reprints per author of each article are furnished free of charge; additional copies may be obtained at cost in multiples of 50 .

The Pacific Journal of Mathematics is published quarterly, in March, June, September, and December. The price per volume (4 numbers) is $\$ 12.00$; single issues, $\$ 3.50$. Back numbers are available. Special price to individual faculty members of supporting institutions and to individual members of the American Mathematical Society: $\$ 4.00$ per volume; single issues, $\$ 1.25$.

Subscriptions, orders for back numbers, and changes of address should be sent to Pacific Journal of Mathematics, 103 Highland Boulevard, Berkeley 8, California.

Printed at Kokusai Bunken Insatsusha (International Academic Printing Co., Ltd.), No. 6, 2-chome, Fujimi-cho, Chiyoda-ku, Tokyo, Japan.

\section{PUBLISHED BY PACIFIC JOURNAL OF MATHEMATICS, A NON-PROFIT CORPORATION}

The Supporting Institutions listed above contribute to the cost of publication of this Journal, but they are not owners or publishers and have no responsibility for its content or policies. 


\section{Pacific Journal of Mathematics}

\section{Vol. 11, No. 2 December, 1961}

Tsuyoshi Andô, Convergent sequences of finitely additive measures........

Richard Arens, The analytic-functional calculus in commutative topological algebras..........................................

Michel L. Balinski, On the graph structure of convex polyhedra in

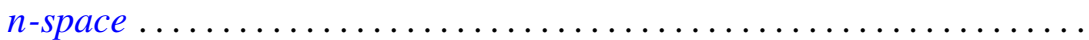

R. H. Bing, Tame Cantor sets in $E^{3}$...

Cecil Edmund Burgess, Collections and sequences of continua in the plane.

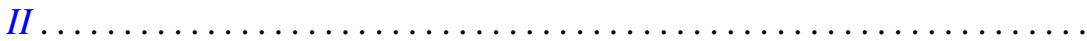

J. H. Case, Another 1-dimensional homogeneous continuum which contains an $\operatorname{arc}$

Lester Eli Dubins, On plane curves with curvature ................. 471

A. M. Duguid, Feasible flows and possible connections .............. 483

Lincoln Kearney Durst, Exceptional real Lucas sequences ................ 489

Gertrude I. Heller, On certain non-linear opeartors and partial differential equations........................................

Calvin Virgil Holmes, Automorphisms of monomial groups

Wu-Chung Hsiang and Wu-Yi Hsiang, Those abelian groups characterized by their completely decomposable subgroups of finite rank ..........

Bert Hubbard, Bounds for eigenvalues of the free and fixed membrane by finite difference methods .........................

D. H. Hyers, Transformations with bounded mth differences. .

Richard Eugene Isaac, Some generalizations of Doeblin's decomposition

John Rolfe Isbell, Uniform neighborhood retracts ..........

Jack Carl Kiefer, On large deviations of the empiric D. F. of vector chance variables and a law of the iterated logarithm...

Marvin Isadore Knopp, Construction of a class of modular functions and forms. II. . .

Gunter Lumer and R. S. Phillips, Dissipative operators in a Banach space....

Nathaniel F. G. Martin, Lebesgue density as a set function ...

Shu-Teh Chen Moy, Generalizations of Shannon-McMillan theorem ...

Lucien W. Neustadt, The moment problem and weak convergence in $L^{2}$

Kenneth Allen Ross, The structure of certain measure algebras...

James F. Smith and P. P. Saworotnow, On some classes of scalar-product algebras.

Dale E. Varberg, On equivalence of Gaussian measures. . 